\title{
Fast and Regularization less Active Contour
}

\author{
Rahul Patel \\ PG Scholar, \\ VTP Department of Electronics \\ \& Communication, \\ CS Patel Institute of \\ Technology, Changa, Gujarat, \\ India.
}

\author{
Hiren Mewada \\ Associate Professor, \\ VTP Department of Electronics \\ \& Communication, \\ CS Patel Institute of \\ Technology, Changa, Gujarat, \\ India.
}

\author{
Suprava Patnaik, PhD. \\ Head and Associate Professor, \\ Electronics Department, \\ SVNIT, Surat, Gujarat, India.
}

\begin{abstract}
The application of the level set method in image segmentation has been very popular due to its capability of automatically handling changes in topology. However, a re-initialization procedure, which leads to expensive computation, is required in the traditional level set method to keep the level set function as a signed distance function to its interface. A method based on Gaussian filtering and binary level set is proposed for the level set function of region based active contour model (ACM). The proposed level set method is integrated with the global region based Chan-Vese (C-V) ACM for image segmentation. The proposed method can, not only ensure the smoothness of the level set function by Gaussian filtering, but also eliminate the requirement of re-initialization, which is very computationally expensive task. The level set function can also be easily initialized as a binary function, which is more efficient to construct practically than the widely used signed distance function (SDF). Moreover, as the proposed scheme allows using larger time step than what can be used with the standard $\mathrm{C}-\mathrm{V}$ model, it is tremendously faster than standard C-V model. Finally, the proposed algorithm can be efficiently implemented by the simple finite difference scheme. Experimental results on synthetic and real images shows that the proposed method is more efficient in terms of computational time and accuracy than global region based $\mathrm{C}-\mathrm{V}$ active contour model.
\end{abstract}

\section{Keywords}

Image segmentation; active contour; level-set method; reinitialization; Gaussian filtering.

\section{INTRODUCTION}

Image segmentation is an important step in image analysis processes. Image segmentation in general has been studied extensively in the past decades. A well-established class of methods are ACMs [1, 2], which have been widely used in image segmentation with promising results since proposed by Kass et al. [6]. The models can achieve sub-pixel accuracy, provide closed and smooth contours/surfaces and can also be treated as automatic segmentation. The existing active contour models can be broadly categorized into two classes: edge-based models and region-based models.

Edge based ACMs [3, 4, 8] relay on gradient of input image for stopping the evolving contour on the boundaries of desired objects. Usually, a stopping function is used to attract the contours to the desired boundaries. Region-based ACMs utilize the image statistical information to construct constraints, and have many advantages over edge-based active contour models. The most important being that as they do not use the image gradient information, they can successfully segment objects
With weak, blurred or even without boundaries. Moreover they can also segment objects from noisy images. One of the most popular region-based active contour models is the $\mathrm{C}-\mathrm{V}$ model [5], which has been successfully used in binary phase segmentation with the assumption that each image region, is statistically homogeneous.

This paper addresses the issues and relevant solution (as described in later section) related to use of level set method in image segmentation. The level set method, first introduced by Osher and Sethian [10] employs the interface of a level set function, i.e., the zero level set, to represent the object boundary in image segmentation. The application of the level set method in image segmentation has been very popular due to its capability of automatically handling changes in topology. However, a re-initialization procedure, which leads to expensive computation, is required in the traditional level set method [11] to keep the level set function as a SDF to its interface.

This paper present the use of global region based $\mathrm{C}-\mathrm{V}$ model [5], where the regularization of level set function is performed using Gaussian kernel filtering term (as discussed in section 4 and [16]) and after each iteration, the level set function is binaries in two level function. The main advantage of the proposed level set scheme for region based active contour models is that the time inefficient re-initialization step required for efficient level set evolution can be totally avoided. Moreover, due to use of Gaussian smoothing, the length penalizing term required for smoothing level set function, can also be removed. This in turn drastically reduces computation time and complexity found in the global C-V model [5]. Moreover due to binarization of level set after each iteration, partial amount of local information is available which helps to identify objects with very weak boundary where standard C-V model [5] fails. In addition to the above advantages, the proposed method also allows initializing the level set as a simple binary function having different sign inside and outside the contour instead of general SDF $[11,12]$. This in turn reduces the complexity present in realization of SDF.

This paper is organized as follows: Section 2 describes the standard C-V model [5] and re-initialization. Section 3 describes proposed level set scheme for region based active contour models. Experimental results are presented in section 4. Finally, concluding remarks in section 5. 


\section{BACKGROUND}

\subsection{Chan-Vese (C-V) ACM}

The C-V model [5] is based on pricewise constant approximation of Mumford-Shah energy functional [9]. For an Image $I(x, y)$ in the image domain $\Omega$, Chan and Vese proposed the fitting energy as shown in (1), where $c_{1}$ and $c_{2}$ are the average intensity inside and outside of the contour $C$, respectively and $\lambda_{1}$ and $\lambda_{2}$ are positive constants. As the two terms in (1) are calculated over entire image domain, C-V model [5] is known as global region based active contour model.

$$
\begin{aligned}
E\left(c_{1}, c_{2}, C\right)= & \lambda_{1} \int_{\text {inside }(C)}\left|I(x, y)-c_{1}\right|^{2} d x d y \\
& +\lambda_{2} \int_{\text {outside }(C)}\left|I(x, y)-c_{2}\right|^{2} d x d y
\end{aligned}
$$

Chan and Vese have added regularization terms like the length of the contour $C$ and area of the region inside $C$. The length of the evolving contour $C$ controls the smoothness of the evolving contour $C$ and further avoids the occurrence of small isolated regions in the final segmentation. The area term control the propagation speed and direction of the evolving contour $C$. Therefore the energy functional becomes

$$
\begin{aligned}
E\left(c_{1}, c_{2}, C\right)= & \mu \text { Length }(C)+v \text { Area }(C) \\
& +\lambda \int_{1} \int_{\text {inside }(c)}\left|I(x, y)-c_{1}\right|^{2} d x d y \\
& +\lambda_{2} \int_{\text {outside }(c)}\left|I(x, y)-c_{2}\right|^{2} d x d y
\end{aligned}
$$

where $\mu, v, \lambda_{1}$ and $\lambda_{2}$ are constants. Generally we take $\lambda_{1}=\lambda_{2}=1$ and $v=0$.

To minimize the energy in (2), the level set method proposed in $[10,11]$ is used. The level set formulation for the $\mathrm{C}-\mathrm{V}$ model is as shown in (3),

$$
\begin{aligned}
& E\left(c_{1}, c_{2}, \phi\right)=\mu \int_{\Omega} \delta_{\varepsilon}(\phi(x, y))|\nabla \phi(x, y)| d x d y \\
& \quad+v \int_{\Omega} H_{\varepsilon}(\phi(x, y)) d x d y \\
& \quad+\lambda_{1} \int_{\Omega}\left|I(x, y)-c_{1}\right|^{2} H_{\varepsilon}(\phi(x, y)) d x d y \\
& \quad+\lambda_{2} \int_{\Omega}\left|I(x, y)-c_{2}\right|^{2}\left(1-H_{\varepsilon}(\phi(x, y))\right) d x d y
\end{aligned}
$$

where $H_{\varepsilon}(z)$ and $\delta_{\varepsilon}(z)$ are slightly regularized versions of 1-D Heaviside function $H(z)$ and direct delta function $\delta(z)$ respectively.

Minimizing the energy in (3) by using the gradient descent method and representing the contour $C$ with zero level set, they obtain the following variational formulation

$$
c 1=\frac{\int_{\Omega} I(x, y) H_{\varepsilon}(\phi(x, y)) d x d y}{\int_{\Omega} H_{\varepsilon}(\phi(x, y)) d x d y}
$$

$$
c 2=\frac{\int_{\Omega} I(x, y)\left(1-H_{\varepsilon}(\phi(x, y))\right) d x d y}{\int_{\Omega}\left(1-H_{\varepsilon}(\phi(x, y))\right) d x d y}
$$

$$
\frac{\partial \phi}{\partial t}=\delta_{\varepsilon}(\phi)\left[\mu \kappa-v-\lambda_{1}(I-c 1)^{2}+\lambda_{2}(I-c 2)^{2}\right](6)
$$

where $\kappa$ is the Euclidean curvature of the contour $C$.

\subsection{Re-initialization}

In the traditional level set methods, the level set function is initialized to be a SDF to its interface in order to prevent it from being too steep or flat near its interface, and because the existing re-initialization schemes were not able to re-initialize the level set function, if the initial level set function is significantly different from a signed distance function. Unfortunately, many existing re-initialization methods [11, 12] have an undesirable side effect of moving the zero level set away from its interface. Furthermore, it is difficult to decide when and how to apply the re-initialization. In addition, reinitialization is a very computationally expensive operation (consumes approximately $90 \%$ of total execution time as shown in section 4). Thus, re-initialization is not only, time consuming operation, but it also affects the accuracy of final segmentation. To overcome this difficulty, Sussman and Fatemi in [15] proposed a method to re-initialize the level set function by solving the following partial difference equation:

$$
\frac{\partial \phi}{\partial t}=\operatorname{sign}\left(\phi_{0}\right)(1-|\nabla \phi|)
$$

where $\phi_{0}$ is the function to be re-initialized, and $\operatorname{sign}\left(\phi_{0}\right)$ is the sign function. When the steady state of Eq. (7) is reached, $\phi$ will be a distance function with the same zero level set as $\phi_{0}$, despite whether $\phi_{0}$ is a distance function or not. This is commonly known as the standard re-initialization procedure. Chan and Vese in [5] used the above equation to re-initialize the level set function.

Another solution to avoid re-initialization of level set is proposed by $\mathrm{Li}$ et al. in [8]. They proposed to integrate and solve a length penalty term as shown in (8), along with the minimization energy. This term force the level set function to be close to a signed distance function. Actually, this penalty term is more like a metric which characterizes how close a function is to a signed distance function.

$$
P(\phi)=\int_{\Omega} \frac{1}{2}(|\nabla \phi(x, y)|-1)^{2} d x d y
$$

However, still it requires to be solved the term in (8) and could not completely avoid re-initialization of level set function in the active contour model.

\section{PROPOSED MODEL}

As reinitialization is time consuming process, use of smoothing the function and binarization process is proposed to eliminate this reinitialization of level set function. Binarization of level set function involves image local information in the evolution and help in local level segmentation process. So this binarization step can be avoided if global segmentation is required.

To avoid the reinitialization, first level set function is initialized as constant binary function having positive constant inside the 
contour and negative constant outside the contour. In equation (6), $\kappa=\operatorname{div}(\nabla \phi /|\nabla \phi|)$, the Euclidean curvature term, in which level set function $\phi$ is initialized as SDF which satisfies $|\nabla \phi|=1[11]$ and it is regularized using Laplacian of it. But according to the theory of scale space in [13] and in [14], evolution of Laplacian of function is equivalent to Gaussian filtering of initial condition of level set function, where sigma of Gaussian function pay role equivalent to the $\mu$ in Equation

(6). Thus in Equation (6), the curvature term $\kappa$ can be avoided with Gaussian smoothing of level set function. Hence the equation 6 in the proposed model is simplified as

$$
\frac{\partial \phi}{\partial t}=\delta_{\varepsilon}(\phi)\left[-\lambda_{1}(I-c 1)^{2}+\lambda_{2}(I-c 2)^{2}\right]
$$

In addition to completely eliminating re-initialization by Gaussian Smoothing and binaryizing the level set, the level set function no longer required to be strictly initialized as a signed distance function. The level set function can be initialized to constants, which have different signs inside and outside the contour. This is very simple to implement in practice compared to signed distance function. In this paper, we initial level set function $\phi_{0}$ simply as:

$$
\phi_{0}=\left\{\begin{array}{lr}
a, & (x, y) \in \Omega_{0}-\partial \Omega_{0} \\
0, & (x, y) \in \partial \Omega_{0} \\
-a, & (x, y) \in \Omega-\partial \Omega_{0}
\end{array}\right.
$$

where $a>0$ is a constant , $\Omega_{0}$ is the subset of the image domain $\Omega$ and $\partial \Omega_{0}$ is the boundary of $\Omega_{0}$.

Finally we implemented the proposed method in an iterative procedure, which consists of the following steps:

(1) Initialize the level set function, $\phi$ as per (10).

(2) Evolve the level set function $\phi$ according to (9).

(3) Smooth the level set function by a Gaussian filter, $G_{\sigma}$ , i.e. $\phi^{\prime}=\phi * G_{\sigma}$

(4) Let $\phi$ take 1 , if $\phi^{\prime} \geq 0$; otherwise let $\phi$ take -1 , if $\phi^{\prime}<0$. Based on result either stop evolution or go to step 2 to continue evolution.

\section{EXPERIMENTAL RESULTS}

In this section, we validated our model by using it on a number of synthetic, natural and medical images. For each image, we conducted the experiments to compare the performance of the proposed Gaussian smoothing and binary level set method based $\mathrm{C}-\mathrm{V}$ method with that of the standard $\mathrm{C}-\mathrm{V}$ active contour [5] for image segmentation. The algorithms were implemented in MATLAB 7.6 on a computer with Intel Core i3 with $2.4 \mathrm{GHz}$ CPU, 3GB RAM and Windows7 64-bit operating system. We also highlight in this section, the effects of different weightage parameters, which can be useful for efficient and accurate image segmentation. Moreover, we also show that the proposed level set based C-V model is less initial contour placement dependent. Results are also shown to prove the improvement of the proposed level set based C-V over standard C-V [5] in terms of segmentation accuracy other than computational time.

As stated earlier, the proposed model utilizes the image statistical information for curve evolution and hence it's less sensitive to noise and weak object boundary. Figure 1 show the result obtained with both, the proposed method and standard C$\mathrm{V}$ method [5]. For standard C-V method, the evolution of the traditional level set converged in $\mathbf{8 6 0}$ iterations and took $\mathbf{1 1 . 9 5}$ seconds (Table 1), whereas with the proposed level set scheme, the evolution converged in $\mathbf{1 0}$ iterations and took $\mathbf{0 . 0 4}$ seconds (Table 1). Moreover based on segmentation accuracy, the proposed method gives result similar to that obtained with standard C-V method [5].
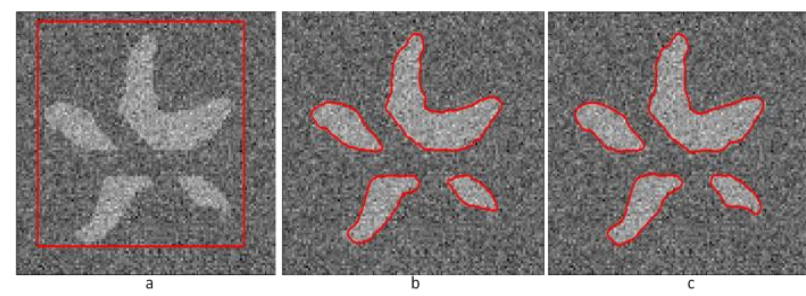

Figure 1: Verification of our method similar to standard C$\mathrm{V}$ method for noisy image (a) Original image with initial contour (b) Result with standard C-V method [5] (c) Result with the proposed method

Figure 2 also shows the results obtained with that of the proposed method and standard C-V method [5]. Again based on the numerical values shown in Table 1 and the results presented in Figure 2(b \& c), we can conclude that the proposed method shows a dramatic reduction in computation time with same amount of segmentation accuracy. Next we test the proposed model on medical image. Figure 3 shows the ability of the proposed method to generate interior contour as generated by standard C-V model (Figure 3(b)).
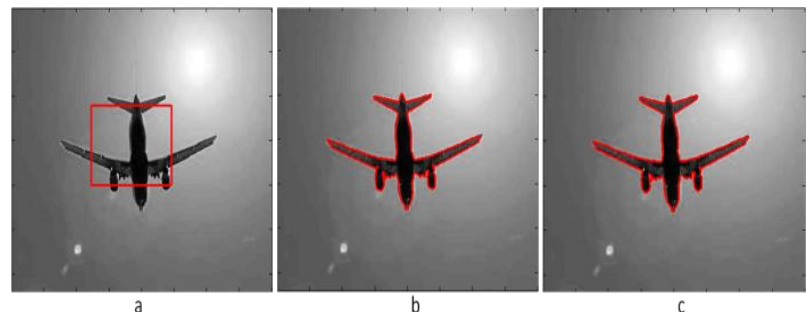

Figure 2: Verification of our method similar to standard C$V$ method (a) Original image with initial contour (b) Result with standard C-V method [5] (c) Result with the proposed method

Figure 4 shows the result of the proposed method for various initializations. This proves the robustness of the described method to initial contour placement. As shown in Figure 4(a), even if the initial contour has been initialized far away from the desired objects in the background with no part of object present inside the contour, we get successful result. Figure 4(b) shows the ability of the proposed level set method to evolve outwards and detect desired objects. Figure 4(c) shows that even if the initial contour encompasses one of the multiple objects present in the input image, other object can be successfully identified. This shows that the proposed level set scheme support evolution of new contours to detect multiple objects. However, with different position of initial contour, the execution time of the proposed method varies by a very minute amount, just equal to nothing. However, for standard C-V method [5], the execution time varies tremendously with different initial contour 
positions. For different initial contours, shown in Figure 4, the execution time was same as that required with the initial contour shown in Figure 1(a) (0.04 seconds).
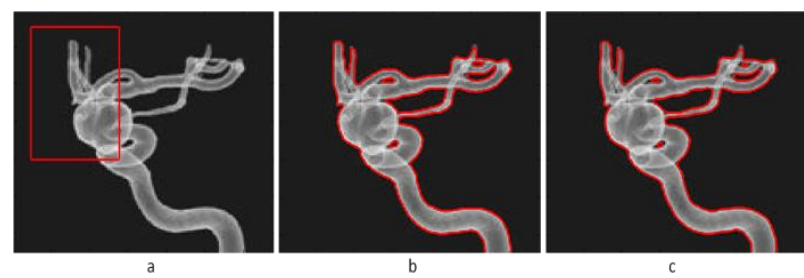

Figure 3: Verification of our method similar to standard C$\mathrm{V}$ method for medical image (a) Original image with initial contour (b) Result with standard C-V method [5] (c) Result with the proposed method
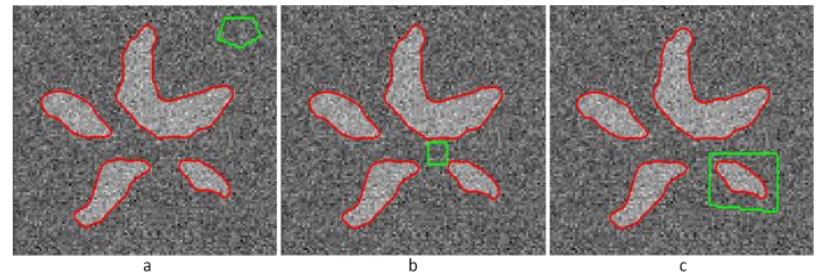

Figure 4: Initial contour placement independence of the proposed method. Figures from (a) to (c) shows different initial contours (in green) and the final contours (in red) using proposed method based $\mathrm{C}-\mathrm{V}$ model.

$\lambda_{1}$ and $\lambda_{2}$ are the coefficients that controls the weightage of proportion of region inside and outside the contour during contour evolution. Therefore relative balance between ${ }^{\lambda_{1}}$ and $\lambda_{2}$ $\lambda_{2}$ determines which side i.e. inside or outside, has higher importance during energy minimization. For most of the images, both these parameters should be selected equal. However, these parameters should be selected properly if we need to segment blurred images. For example, if one wishes to enclose the inside part of blurred object then $\lambda_{1}>>\lambda_{2}$ will insure that (Figure 5(a)) and if one wishes to completely enclose the blurred object, then taking $\lambda_{1}<<\lambda_{2}$ will ensure it (Figure 5(c)). Figure 5(b) shows the result for $\lambda_{1}=\lambda_{2}$.

Next we show the effect of $\sigma$, standard deviation of Gaussian kernel used to regularize the evolving level set after each iteration. Here we show the proof of the statement that $\sigma$ have the same function and effect as the constant $\mu$ used to control the length of the contour $C$ in $\mathrm{C}-\mathrm{V}$ method [5] as shown in equation (6). If we want to detect all or small object individually, then we take $\mu$ small and if we want to detect larger object (e.g. object formed by grouping) and not to detect small object like noise, then take $\mu$ large. The same effect can be seen for small and large values of standard deviation, $\sigma$ of level set regularizing Gaussian kernel. Figure 6(a) shows the result obtained with $\sigma=2$. Here we see that small black circles are effectively identified as individual circles from their adjacent ones. Now with $\sigma=5$, as shown in Figure 6(b), for the same initial contour, grouping of small object take place and are identified as a single large object.

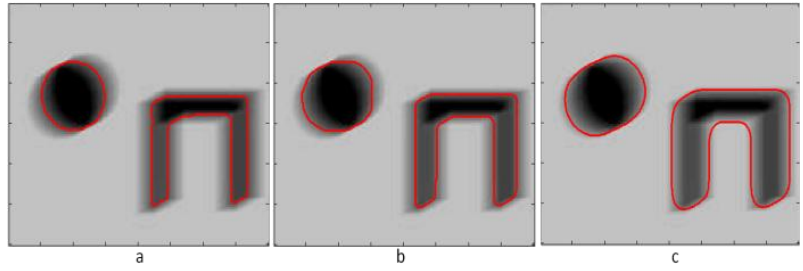

Figure 5: Effect of weighting parameters $\lambda_{1}$ and $\lambda_{2}$ (a)

$$
\lambda_{1}>\lambda_{2} \text { (b) } \lambda_{1}=\lambda_{2} \text { (c) } \lambda_{1}<<\lambda_{2}
$$

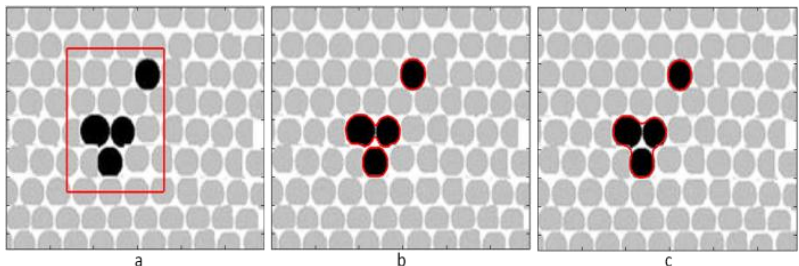

Figure 6: Effect of standard deviation $\sigma$ of regularizing Gaussian Kernel (a) Original image with initial contour (b) Result with small value of $\sigma(\sigma=2)$ (c) Result with large value of $\sigma(\sigma=5)$

Next we show that the binarization of level set function integrate local information along with global, to get accurate results in presence of small amount intensity inhomogeneity. Figure 7 shows the result for natural image which has small amount of intensity inhomogeneity. Here the standard C-V method [5] fails to get over the inhomogeneous part and identify it also as part of the desired object (Figure 7(b)) due to only global dependence (no local information) [7] whereas the proposed method effectively rejects the inhomogeneous part and accurately identify the desired object (Figure 7(c)). Moreover compared to standard $\mathrm{C}-\mathrm{V}$ method, the proposed method is also time efficient, as the accurate result comes in 1.85 seconds in contrary to standard C-V [5], which takes 41.5 seconds for an inaccurate result (Figure 7(b)). If we remove the binarization step and go only with Gaussian smoothing of the level set, we get the result as obtained with standard C-V (Figure 7(b)) with the inhomogeneity part as part of object. This proves that binarization of level set allows integration of local information. However, as Gaussian smoothing avoids reinitialization and regularization, the result comes faster than standard C-V model [5].

Figure 8 again shows the result, where standard C-V model [5] fails and proposed model, gives accurate result. In this figure, we see that, due to the use of binarization of level set, we get some local information in addition to global information, which in turn helped the proposed model to effectively locate the weak edge at the thee of the player (Figure 8(c)), which can't be locate effectively by standard C-V [5] (Figure 8(b)), due to its global dependence, which in turn cause it to get inaccurate result. Moreover the accurate result for the proposed model comes in 11.5 seconds whereas $\mathrm{C}-\mathrm{V}$ [5] gives inaccurate result in 62.1 seconds (Table 1).

In Table 1, the proposed model and standard C-V model [5] are compared relevant to the processing time for the above shown figures along with their size. The table also lists the time taken by re-initialization and regularization terms for the standard C$\mathrm{V}$ model [5]. Last column of Table 1 shows the percentage of the total time, taken by re-initialization and regularization terms in standard C-V model [5]. From the values, it can be seen that nearly $90 \%$ of the total execution time required by standard C$\mathrm{V}$ model [5], is consumed in executing re-initialization and 
regularization terms. Hence we can say that if we can remove re-initialization and regularization terms, then we can drastically reduce the time consumed by any active contour model. By using the proposed level set scheme we totally removed the re-initialization and regularization terms required for efficient evolution of an active contour. Thus as can be seen from the values shown in Table 1 , the time consumed by the proposed model is drastically reduced. The accuracy of the proposed model can be seen directly from the results shown in the corresponding figures.
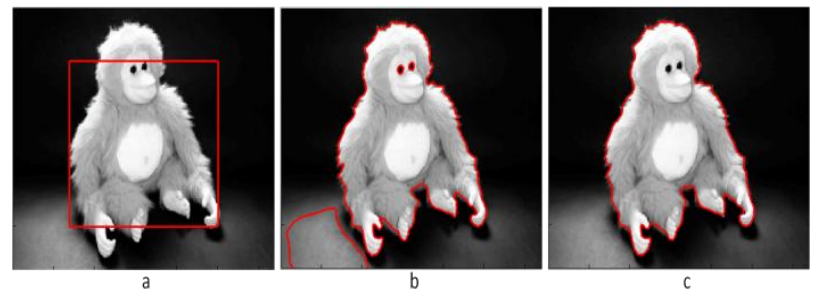

Figure 7: Segmentation of natural images (a) Original image with initial contour (b) Unsuccessful result obtained with standard C-V model [7] (c) Successful segmentation result obtained with proposed model

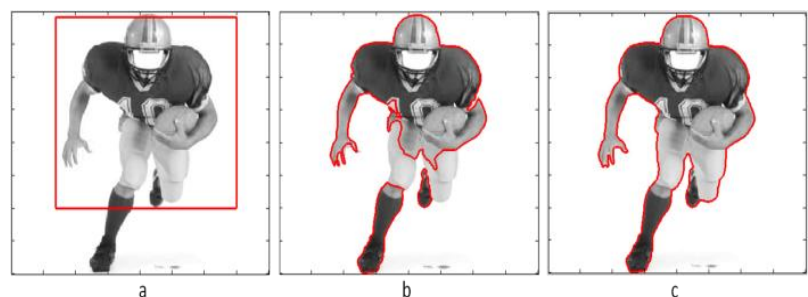

Figure 8: Comparison between tradition C-V [5] and proposed method (a) Original image (b) Unsuccessful segmentation result obtained with standard $C-V$ method [5] (c) Successful segmentation result obtained with proposed method.

\section{CONCLUSION}

This paper proposes a method for completely eliminating reinitialization. Many methods have been proposed to overcome the re-initialization, but they require solving partial difference equation for the same. We presented an approach to eliminate the need of re-initialization and regularization for level set evolution for region based ACMs. In this paper we used Gaussian smoothing and binary level set implementation together to not only eliminate re-initialization but also eliminate the regularization term such as length of the contour. Moreover by binary level set implementation, we incorporated some amount of local information in the evolving level set, which helps to detect weak edges as shown in the experimental results (section 4 above). In addition to this, the proposed method allows the initial level set function to be initialized to constant, which have different signs inside and outside the contour compared to the traditional SDF initialization. This is very simple to implement in practice compared to SDF. Thus the proposed method also reduces the complexity from practical implementation point of view. This paper has integrated the proposed level set based scheme with the standard C-V model, and which founds tremendous reduction in computational time with more accurate results and less initial contour placement dependence, than the original $\mathrm{C}-\mathrm{V}$ active contour model. This paper also highlights the effect of different weightage parameters, which can be useful for efficient and accurate image segmentation.

\section{REFERENCES}

[1] Bakoš, M. 2007. Active Contours and their Utilization at Image Segmentation. In Proc. 5th SlovakianHungarian Joint Symposium on Applied Machine Intelligence and Informatics, Poprad, Slovakia, pp. 313 317.

[2] Blake, A., and Isard, M. 1998. Active Contours, Cambridge, MA: Springer.

[3] Caselles, V., Catte, F., Coll, T., and Dibos, F. 1993. A geometric model for active contours in image processing. Numerische Mathematik, Vol. 66, No. 1, pp. 1-31.

[4] Caselles, V., Kimmel, R., and Sapiro, G. 1997. Geodesic active contour. International Journal of Computer Vision, Vol. 22, No. 1, pp. 61-79.

[5] Chan, T., and Vese, L. 2001. Active contours without edges. IEEE Transaction on Image Processing, Vol. 10, No. 2, pp. 266- 277.

[6] Kass, M., Witkin, A., and Terzopoulos, D. 1988. Snakes: Active contour models. International Journal of Computer Vision, Vol.1, No.4, pp. 321-331.

[7] Lankton, S., and Tannenbaum, A. 2008. Localizing region based active contour. IEEE Transactions on Image Processing, Vol. 17, No. 11, pp. 2029-2039.

[8] Li, C.M., Xu, C.Y., Gui, C.F., and Fox, M.D. 2005 Level set evolution without re-initialization: a new variational formulation. In Proc. IEEE Conference on Computer Vision and Pattern Recognition, San Diego, pp. 430-436.

[9] Mumford, D., and Shah, J. 1989. Optimal approximations by piecewise smooth functions and associated variational problems. Communications on Pure and Applied Mathematics, Vol. 42, No. 5, pp. 577 685.

[10] Osher, S., and Sethian, J.A. 1988. Fronts propagating with curvature dependent speed: algorithms based on Hamilton-Jacobi formulations. Journal of Computational Physics, Vol. 79, No.1, pp. 12-49.

[11] Osher, S., and Fedkiw, R. 2002. Level Set Methods and Dynamic Implicit Surfaces. New York, SpringerVerlag.

[12] Peng, D., Merriman, B., Osher, S., Zhao, H., and Kang, M. 1999. A PDE-based fast local level set method. Journal of Computational Physics, Vol. 155, pp. 410438.

[13] Perona, P., and Malik, J. 1990. Scale-space and edge detection using anisotropic diffusion. IEEE Transaction on Pattern Analysis and Machine Intelligence, Vol. 12, No. 7, pp. 629-640.

[14] Shi, Y., and Karl, W.C. 2005. Real-time tracking using level sets. IEEE Conference on Computer Vision and Pattern Recognition, Vol. 2, pp. 34-41.

[15] Sussman, M., and Fatemi, E. 1999. An efficient, interface preserving level set redistancing algorithm and its application to interfacial incompressible fluid flow. SIAM J. Sci. Comput., Vol. 20, No. 4, pp. 1165-1191.

[16] Zhu, G. P., Zhang, Sh. Q., Zeng, Q. SH., and Wang, Ch. H. 2007. Boundary-based image segmentation using binary level set method. SPIE, OE Letters, Vol.46, No. 5, pp. 1-3. 
Table 1. Processing time (in seconds) taken by the proposed model and standard C-V model [5]

\begin{tabular}{|c|c|c|c|c|c|c|}
\hline $\begin{array}{c}\text { Input } \\
\text { image }\end{array}$ & $\begin{array}{c}\text { Size of } \\
\text { Input } \\
\text { Image }\end{array}$ & $\begin{array}{c}\text { Time taken by } \\
\text { Re-initialization } \\
\text { term in C-V } \\
\text { model [5] }\end{array}$ & $\begin{array}{c}\text { Time taken by } \\
\text { Regularization } \\
\text { term in C-V } \\
\text { model [5] }\end{array}$ & $\begin{array}{c}\text { Total } \\
\text { Time } \\
\text { taken by } \\
\text { C-V } \\
\text { model [5] }\end{array}$ & $\begin{array}{c}\text { Total } \\
\text { Time } \\
\text { taken by } \\
\text { proposed } \\
\text { model }\end{array}$ & $\begin{array}{c}\text { Percentage (\%) of } \\
\text { total time taken by } \\
\text { Re-initialization } \\
+ \\
\text { Regularization terms } \\
\text { in C-V model [5] }\end{array}$ \\
\hline Figure 1 & $112 \times 114$ & 6.5 & 6 & 13.72 & 0.04 & 91.11 \\
\hline Figure 2 & $320 \times 400$ & 18.25 & 17.02 & 38.63 & 0.75 & 91.3 \\
\hline Figure 3 & $130 \times 136$ & 2.5 & 2.3 & 5.13 & 0.15 & 93.56 \\
\hline Figure 7 & $240 \times 320$ & 17.05 & 16.77 & 36.93 & 1.85 & 91.58 \\
\hline Figure 8 & $400 \times 400$ & 32.07 & 29.48 & 67.88 & 11.05 & 90.67 \\
\hline
\end{tabular}

